\title{
EFECTO DE RESIDUOS DE PRODUCCIÓN DE AZÚCAR EN LA ALTURA DEL COMPOST.
}

\section{EFFECT OF SUGAR PRODUCTION RESIDUES AT COMPOST HEIGHT.}

Fabián Alberto Gordillo Manssur, Mgs. Doctorando en Agricultura Protegida (España). Magíster en Gestión de Proyectos (Ecuador). Coordinador de Investigación y docente de la Facultad de Ciencias Agrarias en la Universidad de Guayaquil, Ecuador. fabian.gordillom@ug.edu.ec

Miguel Guzmán Palomino, PhD. Doctor en Ciencias Biológicas (España). Docente del Departamento de Agronomía en la Universidad de Almería, España. mguzman@ual.es

Ivonne Nathaly Casilla Salazar, Mgs. Magíster en Administración Ambiental (Ecuador). Labora en la Dirección de Investigación de la Universidad de Guayaquil, Ecuador. ivonne.casillas@ug.edu.ec

Alexandra Katiuska Rubira Carvache, Mgs. Magíster en Manejo Sustentable de Biorecursos y Medio Ambiente (Ecuador). Labora en la Dirección de Investigación de la Universidad de Guayaquil, Ecuador. rubirac@ug.edu.ec

\section{ARTÍCULO DE INVESTIGACIÓN}

Recibido: 22 de octubre de 2016.

Aceptado: 18 de febrero de 2017.

\section{RESUMEN}

Los desechos de los ingenios azucareros son un problema difícil de manejar, una alternativa para su manejo debe ejercer un control sobre los procesos de biodegradación. 
El estudio se orientó en identificar la influencia del tipo de residuo, fuente de microorganismos y tipo de aireación aplicada, en la descomposición de la materia orgánica (altura) mediante la obtención de un producto por medio del compostaje, colaborando con el mejoramiento de las condiciones edáficas. Para lograr los objetivos propuestos, se elaboraron tres combinaciones de cachaza, bagazo, ceniza, dos fuentes de microorganismos y dos formas de aireación. Las pilas se establecieron bajo un diseño de tres factores, y se valoraron dos veces por semana en campo el parámetro a evaluar; para el análisis de los resultados se utilizaron análisis de varianzas, gráficas y tablas de medias y pruebas de múltiples rangos con un $95 \%$ de confianza mediante el método LSD. Los resultados obtenidos muestran que el factor que más relevante es el método de aireación por volteos y sus interacciones con los otros factores. Los tratamientos que presentaron mayor media de altura fueron con tubos estáticos. Además, los materiales se comportaron de manera similar, de acuerdo a la variable microorganismos y residuo compostado.

Palabras clave: residuos azucareros, compostaje, descomposición.

\section{ABSTRACT}

The waste of sugar mills are a difficult problem to handle, an alternative for management must exercise control over the processes of biodegradation. The study was aimed to identify the influence of the type of waste, source of microorganisms and type of aeration applied in the decomposition of organic matter (height) by obtaining a product by composting, collaborating with the improvement of conditions soil. To achieve the proposed goals, three combinations of filter cake, bagasse, ash, two sources of microorganisms and two forms of aeration were developed. The batteries were established under a design of three factors, and the parameter to be evaluated were assessed twice weekly field; for the analysis of variance analysis results, graphs and tables of medium and multiple range tests with $95 \%$ confidence by LSD method we were used. The results show that the most important factor is the method of aeration volteos and their interactions with other factors. The treatments had higher average height were with static tubes. Furthermore, the materials behave similarly, according to the microorganisms variable and composted waste.

Keywords: sugar residues, composting, descomposition. 


\section{INTRODUCCIÓN}

Los serios problemas de contaminación atribuidos al uso excesivo de fertilizantes inorgánicos han incrementado las áreas que sufren procesos degradativos por la disminución de la fracción orgánica de los suelos ante el intenso uso agrícola. Los ingenios azucareros son grandes contaminadores por la gran cantidad de desechos generados en el proceso agroindustrial, estos residuos pueden revalorizarse dando un tratamiento y uso adecuado, transformándose en materiales orgánicos con la ayuda del desarrollo de tecnologías y el aporte de microorganismos para que estos procesos degradativos sean aprovechados por las diversas actividades del ser humano. Este panorama, renueva el interés por el uso y manejo de los sistemas productivos tales como el manejo de las enmiendas agrícolas aplicadas al suelo, para la recuperación, reutilización y/o transformación de los residuos en insumos útiles a los sectores productivos conservando la fertilidad del suelo y siendo amigable con el ambiente. Las enmiendas orgánicas varían en su composición química de acuerdo al proceso de elaboración, duración del proceso, actividad biológica y tipos de materiales que se utilicen. El compost es el producto estabilizado e higienizado que se obtiene de la descomposición biológica oxidativa (aeróbica) de materiales orgánicos frescos de desechos animales y vegetales, en la cual la principal transformación la sufren los carbohidratos y las proteínas; además, consigue las condiciones consideradas letales para patógenos, parásitos, elementos germinativos y asegurar el aprovechamiento de todos los subproductos que genere el proceso. El objetivo del estudio consiste en evaluar la influencia del tipo de residuo o material, fuente de microorganismos y tipo de aireación aplicada, en la descomposición de la materia orgánica (altura) mediante la obtención de un producto controlado, estabilizado e higienizado por medio del compostaje. Como hipótesis de investigación se tiene que: los residuos orgánicos provenientes de los procesos agroindustriales de la elaboración de azúcar de caña contribuyen a una posible enmienda orgánica estable y controlada mediante la elaboración de compost. El compost elaborado por diferentes porcentajes de combinaciones de los residuos azucareros, distintos métodos de aireación y fuentes de microorganismos cumple con el proceso de descomposición de la materia orgánica mediante la valoración de la altura.

\section{REVISIÓN TEÓRICA}

El proceso de degradación biológica de residuos orgánicos está documentado desde el siglo I d.C. (Holgado y col, 1988). Desde entonces, los agricultores han seguido esta 
práctica (degradación natural) utilizando el producto resultante como abono. Los productos así obtenidos no siempre conservaban su potencial nutritivo debido a la falta de control sobre el proceso. Actualmente (Helynen, 2004; Fitzpatrick y col., 2005), el control, tanto del proceso de producción (compostaje) como del producto final (compost) se hace necesario para asegurar una óptima calidad y mínimos costes.

El compostaje se basa en la acción de diversos microorganismos aerobios (Haug, 1993), que actúan de manera sucesiva, sobre la materia orgánica original, en función de la influencia de determinados factores, produciendo elevadas temperaturas, reduciendo el volumen y el peso de los residuos y provocando su humificación y oscurecimiento (Nakasaki, 2005). Durante este proceso se han de controlar los distintos factores que aseguren una correcta proliferación microbiana y, por consiguiente, una adecuada mineralización de la materia orgánica (Cronje y col, 2003). El hecho de que el compostaje sea biooxidativo exige una condición biológica que lo hace diferente de procesos físicos y químicos, así como de aquellos que no se realicen de forma aeróbica. También se ha de diferenciar entre el compostaje, como proceso controlado, de los procesos naturales no controlados que suelen desembocar en anaerobiosis más o menos acusadas. Al estar regidas estas reacciones por los ciclos biológicos de los microorganismos que intervienen en ellas, el compostaje requiere un tiempo mínimo. Por tanto, un compostaje que pretenda la obtención de un producto final útil como fertilizante (material orgánico estabilizado) no se puede dejar transcurrir espontáneamente, sino que en él han de controlarse las variables necesarias para garantizar la total terminación del proceso en un tiempo corto y con unos costes mínimos (Hedegaard y col, 1996; De Bertoldi y col, 1985; Körner y col, 2003).

\section{Clasificación de los residuos.}

La clasificación de los residuos, admite varios enfoques y la consideración de distintos parámetros. Para la clasificación, se consideran entre otros parámetros: origen o actividad emisora, toxicidad y peligrosidad, tamaño, naturaleza química de los materiales emisores, parámetros físico-químicos en general. Toda clasificación tiende a simplificar la realidad y no abarca todos los casos posibles que se generan por la heterogeneidad en la composición de la naturaleza química e interrelaciones directas e indirectas de las actividades generadoras. 
La clasificación por la naturaleza química permite establecer dos categorías de residuos: residuos inorgánicos o abiógenos y residuos orgánicos o biógenos.

Residuos inorgánicos: incluye todos aquellos residuos de origen mineral y sustancias o compuestos sintetizados por el hombre. Dentro de esta categoría se incluyen habitualmente metales, plásticos, vidrios, etc. Desechos provenientes de agrotóxicos, agroquímicos, fitosanitarios y agroveterinarios, son en su mayoría de origen sintético y con un gran efecto residual. Si bien estos residuos requieren un análisis particular y no son objeto de este trabajo, debe considerarse que los mismos representan importantes insumos en los sectores productivos y su efecto residual puede modificar sustancialmente las características y propiedades de los residuos orgánicos.

Residuos orgánicos: se refiere a todos aquellos que tiene su origen en los seres vivos, animales o vegetales. Incluye una gran diversidad de residuos que se originan naturalmente durante el "ciclo vital" como consecuencia de las funciones fisiológicas de mantenimiento y perpetuación o son producto de explotación por el hombre de los recursos bióticos.

\section{Variables del proceso de compostaje.}

Las variables más importantes que afectan a los sistemas de compostaje pueden ser clasificados en dos tipos de parámetros en los que hay que establecer un control: parámetros de seguimiento (aquellos que han de ser medidos, seguidos durante todo el proceso y adecuados, en caso de ser necesario, para que sus valores se encuentren en los intervalos considerados correctos para cada fase del proceso (Jeris y col, 1973)) y parámetros relativos a la naturaleza del sustrato (aquellos que han de ser medidos y adecuados a su valores correctos fundamentalmente al inicio del proceso (Madejón y col, 2001)). Entre los parámetros de seguimiento se encuentran: temperatura, humedad, pH, aireación y espacio de aire libre. Entre los relativos a la naturaleza del sustrato: tamaño de partícula, relaciones $\mathrm{C} / \mathrm{N}$ y C/P, nutrientes, materia orgánica y conductividad eléctrica.

Los valores o intervalos óptimos están influenciados por las condiciones ambientales, el tipo de residuo a tratar y el sistema de compostaje elegido.

Aireación:

Para el correcto desarrollo del compostaje es necesario asegurar la presencia de oxígeno, ya que los microorganismos que en él intervienen son aerobios. Las pilas de compostaje presentan porcentajes variables de oxígeno en el aire de sus espacios libres: la parte más externa contiene casi tanto oxígeno como el aire (18-20\%); hacia el interior el contenido 
de oxígeno va disminuyendo, mientras que el de dióxido de carbono va aumentando, hasta el punto de que a una profundidad mayor de $60 \mathrm{~cm}$ el contenido de oxígeno puede estar entre 0,5 y $2 \%$ (Ekinci y col, 2004).

Una aireación insuficiente provoca una sustitución de los microorganismos aerobios por anaerobios, con el consiguiente retardo en la descomposición, la aparición de sulfuro de hidrógeno y la producción de malos olores (Bidlingmaier, 1996). El exceso de ventilación podría provocar el enfriamiento de la masa y una alta desecación con la consiguiente reducción de la actividad metabólica de los microorganismos (Zhu, 2006).

La aireación es una variable de operación muy importante y la que más incide en los costes de operación, ya que suponen el $32-46 \%$ de los costes totales. La medida de la concentración de oxígeno requiere equipos costosos, pero puede también realizarse de una manera indirecta mediante las medidas de temperatura y humedad (Kulcu y col, 2004).

Durante el proceso de maduración no deben hacerse aportaciones adicionales de oxígeno, ya que una excesiva aireación podría dar lugar a un consumo de los compuestos húmicos formados y a una rápida mineralización de los mismos (Tomati y col., 2000).

Estructura y tamaño de los residuos:

Numerosos materiales pierden rápidamente su estructura física cuando ingresan al proceso de compostaje (por ej. excretas), otros no obstante son muy resistentes a los cambios, tal es el caso de materiales leñosos y fibras vegetales en general. En este caso la superficie de contacto entre el microorganismo y los desechos es pobre, no olvide el carácter osmótrofo de la gran mayoría de las bacterias.

Cuando se presenta una situación de este tipo, por ejemplo disponemos de restos de podas de pequeño diámetro, debemos mezclar estos residuos con otros de diferente estabilidad estructural, de forma tal que aumente la superficie de contacto. Una opción sería la mezcla de estos restos de poda con excretas en proporciones tales que aseguremos una buena $\mathrm{C} / \mathrm{N}$ de entrada.

Ante el caso de no disponer, de excretas u otro material de diferente estructura física, debemos recurrir al procesamiento del mismo para lograr un tamaño adecuado y un proceso rápido. 


\section{MATERIALES Y MÉTODOS}

\section{Especialidades de la materia prima.}

Se utilizaron los residuos azucareros (bagazo, ceniza y cachaza) del Ingenio La Troncal localizado en el cantón La Troncal y se usaron dos fuentes de microorganismos: uno artesanalmente en la hacienda San Humberto ubicada en el Cantón Taura de la Provincia del Guayas y el otro es un producto comercial cuyo nombre es QBio COMPOSTER elaborado por Quality Corporation.

Se establecieron los porcentajes de cada uno de los residuos que conciernen a las tres formulaciones utilizadas, como se muestra en la Tabla 1:

Tabla 1: Caracterización de la materia prima.

Fuente: elaboración propia.

\begin{tabular}{|c|c|c|c|}
\hline Materia Prima & $\%$ Humedad & $\begin{array}{c}\% \\
\text { Cenizas }\end{array}$ & $\begin{array}{c}\% \text { Materia } \\
\text { orgánica }\end{array}$ \\
\hline Bagazo & 46,539 & 4,476 & 48,985 \\
\hline Cachaza & 86,751 & 2,739 & 10,510 \\
\hline Ceniza & 53,903 & 40,139 & 5,958 \\
\hline
\end{tabular}

\section{Formulación de las unidades experimentales.}

De acuerdo a la caracterización de los materiales, se procedió a realizar tres combinaciones entre los materiales y luego, se establecieron las diferentes combinaciones correspondientes a cada tratamiento como se muestra en la Tabla 2.

Tabla 2: Combinación de factores.

\begin{tabular}{|c|c|c|c|}
\hline Tratamiento & $\begin{array}{c}\text { Fuente de } \\
\text { microorganismos }\end{array}$ & Formulación & $\begin{array}{c}\text { Método de } \\
\text { aireación }\end{array}$ \\
\hline T1 & Locales & C1 & Tubos \\
\hline T2 & Locales & C1 & Volteo \\
\hline T3 & Locales & C2 & Tubos \\
\hline T4 & Locales & C2 & Volteo \\
\hline T5 & Locales & C3 & Tubos \\
\hline T6 & Locales & C3 & Volteo \\
\hline T7 & Comerciales & C1 & Tubos \\
\hline T8 & Comerciales & C1 & Volteo \\
\hline
\end{tabular}


Revista Científica ECOCIENCIA

\begin{tabular}{|c|c|c|c|}
\hline T9 & Comerciales & C2 & Tubos \\
\hline T10 & Comerciales & C2 & Volteo \\
\hline T11 & Comerciales & C3 & Tubos \\
\hline T12 & Comerciales & C3 & Volteo \\
\hline T13 & Ninguno & C1 & Tubos \\
\hline T14 & Ninguno & C1 & Volteo \\
\hline T15 & Ninguno & C2 & Tubos \\
\hline T16 & Ninguno & C2 & Volteo \\
\hline T17 & Ninguno & C3 & Tubos \\
\hline T18 & Ninguno & C3 & Volteo \\
\hline
\end{tabular}

Fuente: elaboración propia.

Luego, se realizaron las pilas para el compost bajo un diseño de tres factores, las mismas que tuvieron una medida de ocho metros de largo por 2,5 metros de ancho y 1,5 metros de altura. Para la aplicación de los microorganismos comerciales se tomó la dosis recomendada por el fabricante (4,5g/t); la aplicación de los microorganismos locales se aplicó la dosis normalmente usada por el agricultor $(11 / \mathrm{kg})$. Una vez terminada la instalación de las pilas se colocaron los tubos en los tratamientos que correspondían. Los tubos tuvieron una medida de un metro de largo y fueron colocados en tres lugares de cada pila, los mismos que permanecieron constantes durante el proceso de compostaje. Los tratamientos con el método de aireación de volteo, se realizó el volteo dos veces por semana a cada pila antes de tomar la lectura de los parámetros en estudio.

\section{Muestreo y evaluaciones.}

El parámetro a evaluar es altura, la misma se valoró en la parte media de cada pila con una regla vertical durante todo el proceso de compostaje con frecuencia de dos veces por semana.

\section{ANÁLISIS DE RESULTADOS Y DISCUSIÓN}

Todos los resultados de las variables medidas fueron evaluados estadísticamente mediante ANOVA Multifactorial, tablas y gráficos de media y pruebas de múltiples rangos al 95\% de confianza (STATGRAPHICS CENTURION XVII).

Todos los tratamientos se encuentran relacionados con al menos un tratamiento de acuerdo a sus medias. Además, la altura $(\mathrm{cm})$ registrada en los tratamientos se encuentra en un rango entre $87 \mathrm{~cm}$ a $117 \mathrm{~cm}$. 
Figura 1: Medias por tratamiento en función de la variable Altura.

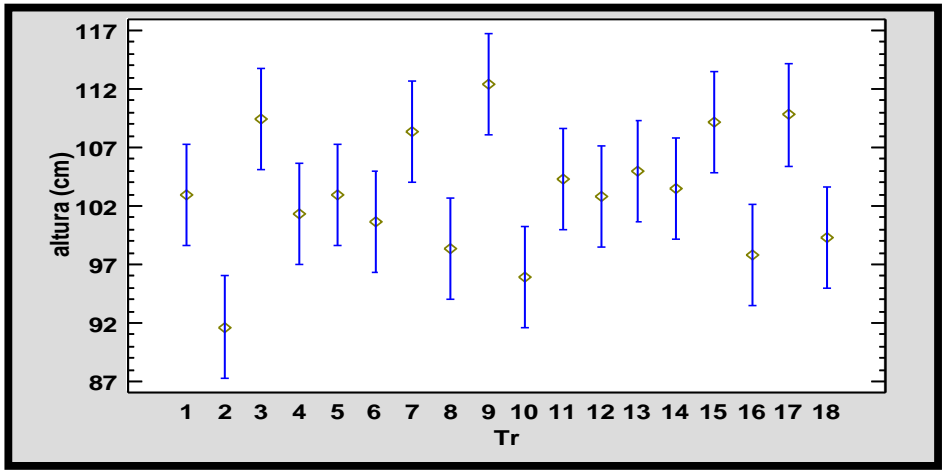

Fuente: elaboración propia.

Tabla 3: Medias por Mínimos Cuadrados para altura $(\mathrm{cm})$ con intervalos de confianza del $95,0 \%$.

\begin{tabular}{|c|l|l|l|l|l|}
\hline & & & Error & Límite & Límite \\
\hline Nivel & Casos & Media & Est. & Inferior & Superior \\
\hline MEDIA GLOBAL & 648 & 103,091 & & & \\
\hline Tratamientos & & & & & \\
\hline 1 & 36 & 102,888 & 3,11917 & 96,774 & 109,001 \\
\hline 2 & 36 & 91,6722 & 3,11917 & 85,5587 & 97,7857 \\
\hline 3 & 36 & 109,475 & 3,11917 & 103,362 & 115,588 \\
\hline 4 & 36 & 101,354 & 3,11917 & 95,2407 & 107,468 \\
\hline 5 & 36 & 102,892 & 3,11917 & 96,7782 & 109,005 \\
\hline 6 & 36 & 100,672 & 3,11917 & 94,5587 & 106,786 \\
\hline 7 & 36 & 108,353 & 3,11917 & 102,239 & 114,466 \\
\hline 8 & 36 & 98,3361 & 3,11917 & 92,2226 & 104,45 \\
\hline 9 & 36 & 112,44 & 3,11917 & 106,327 & 118,554 \\
\hline 10 & 36 & 95,9014 & 3,11917 & 89,7879 & 102,015 \\
\hline 11 & 36 & 104,292 & 3,11917 & 98,1782 & 110,405 \\
\hline 12 & 36 & 102,832 & 3,11917 & 96,7185 & 108,945 \\
\hline 13 & 36 & 104,986 & 3,11917 & 98,8726 & 111,1 \\
\hline 14 & 36 & 103,489 & 3,11917 & 97,3754 & 109,602 \\
\hline 15 & 36 & 109,118 & 3,11917 & 103,005 & 115,232 \\
\hline 16 & 36 & 97,8194 & 3,11917 & 91,706 & 103,933 \\
\hline 17 & 36 & 109,763 & 3,11917 & 103,649 & 115,876 \\
\hline 18 & 36 & 99,3486 & 3,11917 & 93,2351 & 105,462 \\
\hline
\end{tabular}

Fuente: elaboración propia. 
En la Tabla 3, se observa que las dos columnas de la extrema derecha muestran intervalos de confianza del 95,0\% para cada una de las medias. La mayor altura $(\mathrm{cm})$ de acuerdo a la media registrada en los tratamientos, fue la del tratamiento 9 (fuente de microorganismos comerciales, formulación dos y aireación por tubos estáticos) con $112,44 \mathrm{~cm}$, seguido por el tratamiento 17 (Ninguna fuente de microorganismos, formulación tres y aireación por tubos estáticos) con 109,763 cm y tratamiento 15 (Ninguna fuente de microorganismos, formulación dos y aireación por tubos estáticos) con $109,118 \mathrm{~cm}$. Además, en mencionados tratamientos se registraron valores superiores e inferiores, superiores a los demás tratamientos. La menor altura $(\mathrm{cm})$ de acuerdo a la media registrada en los tratamientos, fue la del tratamiento 2 (fuente de microorganismos locales, formulación uno y aireación por volteos) con 91,6722 cm, seguido por el tratamiento 10 (fuente de microorganismos comerciales, formulación dos y aireación por volteos) con $95,9014 \mathrm{~cm}$ y tratamiento 16 (Ninguna fuente de microorganismos, formulación dos y aireación por volteos) con 97,8194cm. Además, en mencionados tratamientos se registraron valores superiores e inferiores, inferiores a los demás tratamientos.

Tabla 4: Análisis de Varianza para altura (cm) - Suma de Cuadrados Tipo III.

\begin{tabular}{|l|l|l|l|l|l|}
\hline \multicolumn{1}{|c|}{ Fuente } & Suma de Cuadrados & GI & Cuadrado Medio & Razón-F & Valor-P \\
\hline EFECTOS PRINCIPALES & & & & & \\
\hline A:Tr & 17905,5 & 17 & 1053,26 & 3,01 & 0,0000 \\
\hline RESIDUOS & 220659, & 630 & 350,253 & & \\
\hline TOTAL (CORREGIDO) & 238565, & 647 & & & \\
\hline
\end{tabular}

Fuente: elaboración propia.

En la tabla 4 puesto que se ha escogido la suma de cuadrados Tipo III (por omisión), la contribución de cada factor se mide eliminando los efectos de los demás factores. Puesto que un valor-P es menor que 0,05 , este factor tratamientos tiene un efecto estadísticamente significativo sobre altura $(\mathrm{cm})$ con un 95,0\% de nivel de confianza.

Tabla 5: Pruebas de Múltiple Rangos para altura (cm) por Tratamientos (Tr).

\begin{tabular}{|l|l|l|l|l|}
\hline $\operatorname{Tr}$ & Casos & Media LS & Sigma LS & Grupos Homogéneos \\
\hline 2 & 36 & 91,6722 & 3,11917 & $\mathrm{X}$ \\
\hline 10 & 36 & 95,9014 & 3,11917 & $\mathrm{XX}$ \\
\hline
\end{tabular}


Revista Científica ECOCIENCIA

\begin{tabular}{|l|l|l|l|l|}
\hline 16 & 36 & 97,8194 & 3,11917 & $\mathrm{XXX}$ \\
\hline 8 & 36 & 98,3361 & 3,11917 & $\mathrm{XXX}$ \\
\hline 18 & 36 & 99,3486 & 3,11917 & $\mathrm{XXX}$ \\
\hline 6 & 36 & 100,672 & 3,11917 & $\mathrm{XXX}$ \\
\hline 4 & 36 & 101,354 & 3,11917 & $\mathrm{XXXX}$ \\
\hline 12 & 36 & 102,832 & 3,11917 & $\mathrm{XXXX}$ \\
\hline 1 & 36 & 102,888 & 3,11917 & $\mathrm{XXXX}$ \\
\hline 5 & 36 & 102,892 & 3,11917 & $\mathrm{XXXX}$ \\
\hline 14 & 36 & 103,489 & 3,11917 & $\mathrm{XXXX}$ \\
\hline 11 & 36 & 104,292 & 3,11917 & $\mathrm{XXXXX}$ \\
\hline 13 & 36 & 104,986 & 3,11917 & $\mathrm{XXXX}$ \\
\hline 7 & 36 & 108,353 & 3,11917 & $\mathrm{XXX}$ \\
\hline 15 & 36 & 109,118 & 3,11917 & $\mathrm{XXX}$ \\
\hline 3 & 36 & 109,475 & 3,11917 & $\mathrm{XX}$ \\
\hline 17 & 36 & 109,763 & 3,11917 & $\mathrm{XX}$ \\
\hline 9 & 36 & 112,44 & 3,11917 & $\mathrm{X}$ \\
\hline
\end{tabular}

Fuente: elaboración propia.

La tabla 5 aplica un procedimiento de comparación múltiple para determinar cuáles medias son significativamente diferentes de otras. Se han identificado 6 grupos homogéneos según la alineación de las X's en columnas. No existen diferencias estadísticamente significativas entre aquellos niveles que compartan una misma columna de X's. Luego, se realizaron pruebas de múltiples rangos y gráficas de medias para determinar qué factores tienen un efecto estadísticamente significativo sobre altura $(\mathrm{cm})$.

Tabla 6: Pruebas de Múltiple Rangos para altura (cm) por DDT.

\begin{tabular}{|l|l|l|l|l|}
\hline$D D T$ & Casos & Media LS & Sigma LS & Grupos Homogéneos \\
\hline 44 & 54 & 86,9815 & 1,14592 & $\mathrm{X}$ \\
\hline 40 & 54 & 87,3333 & 1,14592 & $\mathrm{X}$ \\
\hline 36 & 54 & 88,3889 & 1,14592 & $\mathrm{X}$ \\
\hline 32 & 54 & 94,8704 & 1,14592 & $\mathrm{X}$ \\
\hline 28 & 54 & 95,9444 & 1,14592 & $\mathrm{X}$ \\
\hline 24 & 54 & 99,4259 & 1,14592 & $\mathrm{X}$ \\
\hline 20 & 54 & 102,019 & 1,14592 & $\mathrm{XX}$ \\
\hline 12 & 54 & 104,352 & 1,14592 & $\mathrm{XX}$ \\
\hline
\end{tabular}


Revista Científica ECOCIENCIA

\begin{tabular}{|l|l|l|l|c|}
\hline 16 & 54 & 107,278 & 1,14592 & $X X$ \\
\hline 8 & 54 & 107,685 & 1,14592 & $X$ \\
\hline 4 & 54 & 111,759 & 1,14592 & $X$ \\
\hline 0 & 54 & 151,05 & 1,14592 & $X$ \\
\hline
\end{tabular}

Fuente: elaboración propia.

Esta tabla 6 aplica un procedimiento de comparación múltiple para determinar cuáles medias son significativamente diferentes de otras. Por lo expuesto, se observa que existe una disminución en la altura a medida que transcurren los días de compostaje.

Figura 2: Medias de los factores en función de la variable Altura (,$B$ y C).
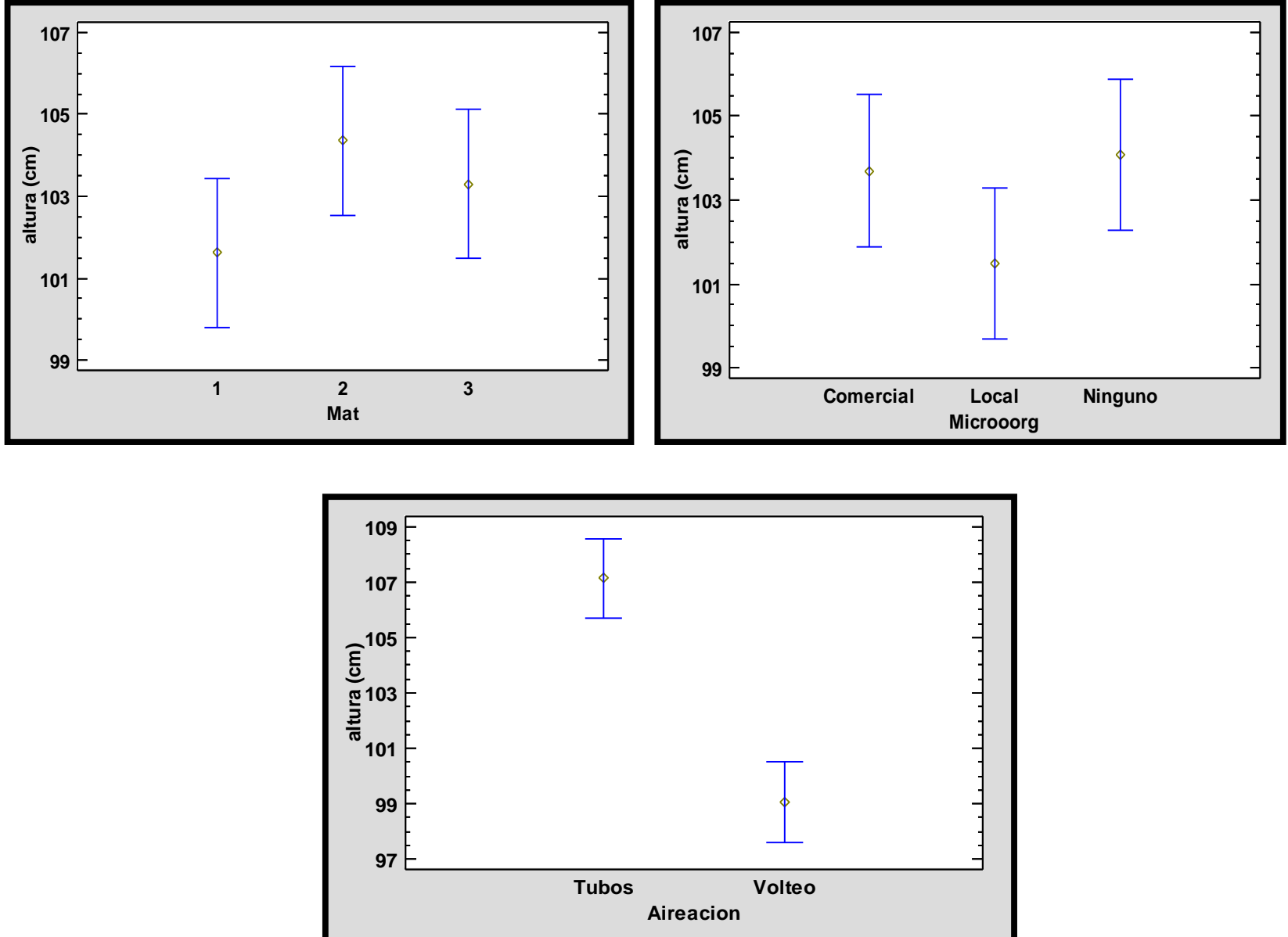

Fuente: elaboración propia.

En la figura $2 \mathrm{~A}, \mathrm{~B}, \mathrm{C}$, se observa que para los factores material y microorganismos todos los tratamientos se encuentran relacionados con al menos un tratamiento de acuerdo a 
sus medias; caso contrario, con el factor aireación que si incide en el comportamiento de la variable altura. Por lo expuesto, se procedió a confirmar lo observado en las gráficas de medias mediante pruebas de múltiples rangos para cada factor.

Tabla 7: Pruebas de Múltiple Rangos para altura en cm. (A, B, C y D).

\begin{tabular}{|l|l|l|l|l|}
\hline Aireación & Casos & $\begin{array}{l}\text { Media } \\
\text { LS }\end{array}$ & $\begin{array}{l}\text { Sigma } \\
\text { LS }\end{array}$ & $\begin{array}{l}\text { Grupos } \\
\text { Homogéneos }\end{array}$ \\
\hline Volteo & 324 & 99,0472 & 1,04364 & $\mathrm{X}$ \\
\hline Tubos & 324 & 107,134 & 1,04364 & $\mathrm{X}$ \\
\hline
\end{tabular}

\begin{tabular}{|l|l|l|l|l|}
\hline Microoorg & Casos & $\begin{array}{l}\text { Media } \\
\text { LS }\end{array}$ & $\begin{array}{l}\text { Sigma } \\
\text { LS }\end{array}$ & $\begin{array}{l}\text { Grupos } \\
\text { Homogéneos }\end{array}$ \\
\hline Local & 216 & 101,492 & 1,30625 & $\mathrm{X}$ \\
\hline Comercial & 216 & 103,692 & 1,30625 & $\mathrm{X}$ \\
\hline Ninguno & 216 & 104,087 & 1,30625 & $\mathrm{X}$ \\
\hline
\end{tabular}

\begin{tabular}{|l|l|l|l|l|l|}
\hline \multicolumn{2}{|l|}{ Contraste } & Sig. & Diferencia & + +- Límites \\
\hline \multicolumn{2}{|l|}{ Tubos - Volteo } & $*$ & 8,08673 & 2,89278 \\
\hline Mat & Casos & $\begin{array}{l}\text { Media } \\
\text { LS }\end{array}$ & $\begin{array}{l}\text { Sigma } \\
\text { LS }\end{array}$ & $\begin{array}{l}\text { Grupos } \\
\text { Homogéneos }\end{array}$ \\
\hline 1 & 216 & 101,621 & 1,30632 & $\mathrm{X}$ \\
\hline 3 & 216 & 103,3 & 1,30632 & $\mathrm{X}$ \\
\hline 2 & 216 & 104,351 & 1,30632 & $\mathrm{X}$ \\
\hline
\end{tabular}

Fuente: elaboración propia.

La tabla 7 (A, B, C, D) muestra la aplicación de un procedimiento de comparación múltiple para determinar cuáles medias son significativamente diferentes de otras. Por lo expuesto, se observa que no hay diferencias estadísticamente significativas entre cualquier par de medias, con un nivel del 95,0\% de confianza. Además, se realizó un gráfico que represente el comportamiento de la descomposición del material compostado en función de los días de establecidos los tratamientos. 
Figura 3: Medias del material en función de los días de establecimiento.

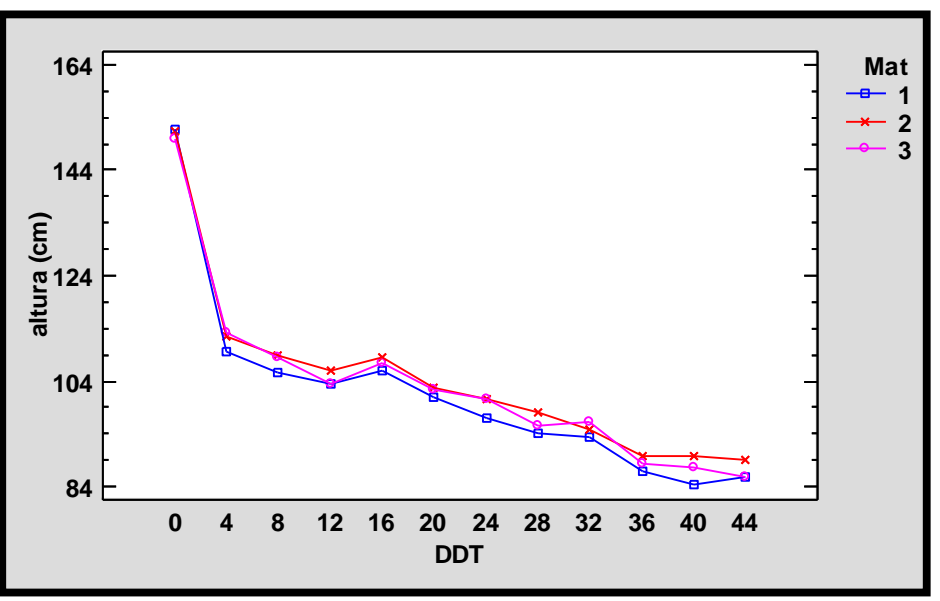

Fuente: elaboración propia.

En la figura 3, se observa que todos los materiales se comportaron de manera similar, con una disminución en la altura de las pilas de compostaje producto de la descomposición, realizado por bacterias aeróbicas termófilas y las temperaturas alcanzadas son superiores a los $60^{\circ} \mathrm{C}$.

\section{CONCLUSIONES}

El factor más relevante que interviene en el proceso de disminución de altura que indica la descomposición del material compostado, es el método de aireación por volteos.

No se observaron diferencias significativas entre los materiales compostados y las fuentes de microorganismos referente a la variable en estudio.

El proceso de compostaje presentó una fase de mineralización de la materia orgánica expresado en la disminución de la altura de las pilas de compost al finalizar el proceso.

\section{REFERENCIAS BIBLIOGRÁFICAS}

Agreda, G. y Deza, M. (2010). Factores que condicionan el proceso de compostaje. Recuperado, enero del 2010. http://www.uam.es.

Bari, Q. y Koenig, A. (2001). Effect of air recirculation and reuse on composting of organic solid waste. Resources, Conservation and Recycling, 33(2), 93-111. http://doi.org/10.1016/S0921-3449(01)00076-3. 
Barrena, R. (2006). Compostaje de residuos sólidos orgánicos. Aplicación de técnicas respirométricas en el seguimiento del proceso. Memoria de Tesis. Universitat Autònoma de Barcelona-Escola Universitària Politècnica del Medi Ambient.

Benzing, A. (2001). Agricultura Orgánica- fundamentos para la región andina. Neckar Verlag, Alemania. p.682.

Bidlingmaier, W. (1996). Odour Emissions from Composting Plants. In M. de Bertoldi, P. Sequi, B. Lemmes, y T. Papi (Eds.), The Science of Composting (pp. 71-80). Dordrecht: Springer Netherlands. http://doi.org/10.1007/978-94-009-1569-5_8

Brewer, L. y Sullivan, D. (2003). Maturity and stability evaluation of composted yard trimmings. Compost Science Y Utilization, 11(2), 96-112.

Cárdenas, G. y B, Guzmán. (1983). Capacidad contaminante de las cachazas producidas por los ingenios azucareros de Tucumán. Argentina. Rev. Ind. Agr. de Tucumán., 60(1): p.59-67.

Cayuela, M. Sánchez-Monedero, M. y Roig, A. (2006). Evaluation of two different aeration systems for composting two-phase olive mill wastes. Process Biochemistry, 41(3), 616-623.

Chávez, E. (2009). Determinación de la calidad de biofertilizantes líquidos y estudio del potencial para la inhibición de Micospharela fijiensis (Morelet). Tesis de grado (Ing. Agropecuario) Guayaquil, Ecuador. p.18-25.

Edufuturo. (2006). La contaminación. http://www.edufuturo.com. Consultado, enero 2010.

Instituto Nacional de Normalización (INN). (2005). Norma para valorar la calidad de un compost. Servicio agrícola ganadero. Chile. Recueprado, enero 2010. www.inn.cl.

Liang, C. Das, K. y McClendon, R. (2003). The influence of temperature and moisture contents regimes on the aerobic microbial activity of a biosolids composting blend. 
Bioresource Technology, 86(2), 131-137. http://doi.org/10.1016/S09608524(02)00153-0

Madejón, E. López, R. Murillo, J. y Cabrera, F. (2001). Agricultural use of three (sugarbeet) vinasse composts: effect on crops and chemical properties of a Cambisol soil in the Guadalquivir river valley (SW Spain). Agriculture, Ecosystems y Environment, 84(1), 55-65. Recuperado en septiembre de 2016. http://www.sciencedirect.com/science/article/pii/S0167880900001912.

Márquez, P. Díaz Blanco, M. y Cabrera, F. (2005). Factores que afectan al proceso de Compostaje, $16 . \quad$ Recuperado, agosto de 2016. http://digital.csic.es/bitstream/10261/20837/3/Factores que afectan al proceso de compostaje.pdf

Mato, S. Mariño, F. y Domínguez, J. (2004). La estrategia del proceso de compostaje como elemento fundamental para la obtención de un compost de calidad. Microbiology, (figura 1), 205-206.

Moreno, J. y Moral, R. (2007). Compostaje. Ediciones Mundi-Prensa, Madrid, España. p. 93-285.

Peña, A. (1999). Los residuos industria azucarera (CEA) y el medio ambiente. Recuperado, enero 2010. http://www.bvsde.paho.org.

Pravia, M. (1999). Guía de Compostaje, Proyectos Residuos Rosario, Argentina. p.27 -34.

Suquilanda, M. (1995). Agricultura orgánica, alternativa tecnológica del futuro. p.172-208.

\section{Licencia Creative Commons}

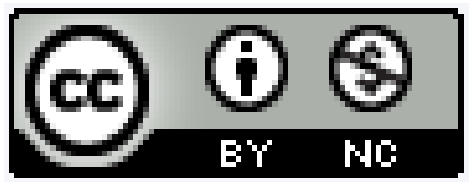

Revista Científica ECOCIENCIA está bajo una Licencia Creative Commons Atribución-NoComercial 4.0 Internacional. 